\title{
Delirium after primary percutaneous coronary intervention in aged individuals with acute ST-segment elevation myocardial infarction: A retrospective study
}

\author{
SHENG LI, XIAO-HONG ZHANG, GEN-DONG ZHOU and JIAN-FEI WANG \\ Department of Cardiology, The Third Affiliated Hospital of Anhui Medical University, Hefei, Anhui 230061, P.R. China
}

Received July 25, 2018; Accepted December 31, 2018

DOI: $10.3892 /$ etm.2019.7398

\begin{abstract}
The present prospective study aimed to investigate the incidence and risk factors of delirium after primary percutaneous coronary intervention (PCI) in older adults with acute ST-segment elevation myocardial infarction (STEMI). A total of 111 patients (age, $\geq 65$ years) with acute STEMI following primary PCI were included in the present study. Neurocognitive testing was performed using the Mini-mental State Examination on the first day of hospitalization. Post-operative delirium was assessed using the Confusion Assessment Method for the Intensive Care Unit within the first four post-operative days. A total of 32 patients (28.8\%) developed delirium after primary PCI. The independent predictors of delirium were older age [odds ratio $(\mathrm{OR})=1.192,95 \%$ confidence interval $(\mathrm{CI})=1.07-1.328, \mathrm{P}=0.001]$, living alone $(\mathrm{OR}=4.827,95 \% \mathrm{CI}=1.315-17.725, \mathrm{P}=0.018)$, history of alcohol abuse $(\mathrm{OR}=3.875,95 \% \mathrm{CI}=1.168-12.857, \mathrm{P}=0.026)$, longer duration of primary PCI $(\mathrm{OR}=1.152,95 \% \mathrm{CI}=1.077-1.232$, $\mathrm{P}<0.001$ ) and post-operative pain (current pain; $\mathrm{OR}=7.663$, 95\% CI=1.432-41.02, $\mathrm{P}=0.017$ ). Compared to the patients without delirium, the participants who developed delirium had longer hospital stays and a higher rate of re-admission within 30 days after discharge. The mortality within one year after discharge (one-year mortality) was similar between patients with and without delirium. In conclusion, older patients (age, $\geq 65$ years) with acute STEMI are at a relatively high risk of delirium following primary PCI. Higher age ( $\geq 65$ years), living alone, history of alcohol dependence, longer length of primary PCI (>50 min) and post-operative pain (current pain) were determined to be risk factors for delirium after primary PCI in the present cohort.
\end{abstract}

Correspondence to: Dr Xiao-Hong Zhang, Department of Cardiology, The Third Affiliated Hospital of Anhui Medical University, 390 Huaihe Road, Luyang, Hefei, Anhui 230061, P.R. China

E-mail: xiaohongzhangdr@163.com

Key words: ST-segment elevation, myocardial infarction, percutaneous coronary intervention, delirium, incidence, risk factors

\section{Introduction}

Post-operative delirium is an acute neuropsychiatric clinical syndrome primarily characterized by disturbances in memory, thought, behaviour, perception, orientation and consciousness. This syndrome is a common and unrecognized condition after surgery in aged individuals (1). Delirium is not only a manifestation of clinical syndromes, but also associated with increased mortality (2), longer hospital stay, as well as a poorer physical performance and social functioning (3). Furthermore, $25 \%$ of individuals aged $\geq 65$ years have been reported to experience delirium during hospitalization $(4,5)$. Given the projected aging of the population, a growing number of elderly patients may undergo surgery in the future. Therefore, post-operative delirium is likely to be more prevalent, and much attention must be paid to post-operative delirium in elderly patients.

The therapy of coronary heart disease mainly includes coronary artery bypass graft surgery and percutaneous coronary intervention (PCI). For coronary heart disease patients, post-operative delirium has been the most extensively investigated after coronary artery bypass graft surgery $(6,7)$. The incidence of delirium in individuals with acute ST-segment elevation myocardial infarction (STEMI) within $12 \mathrm{~h}$ after primary PCI has not been determined in Chinese subjects, although primary PCI is increasingly common in older adults with acute STEMI. Thus, there is probably a large unrecognized population of older patients with STEMI who have developed delirium after primary PCI. The major objective of the present study was to investigate the incidence and risk factors of delirium in older adults (age, $\geq 65$ years) with STEMI after primary PCI.

\section{Patients and methods}

Patients. The present prospective observational study was performed at the Department of Cardiology of the Third Affiliated Hospital of Anhui Medical University (Hefei, China), and the participants were enrolled between February 2014 and February 2016. The follow-up was performed at one year after discharge. In the present study, a diagnosis of acute STEMI was reached based on the following criteria: i) Chest pain that lasted for $>30 \mathrm{~min}$, and was not relieved by sublingual glyceryl trinitrate; ii) $>0.1 \mathrm{mV}$ of ST-segment elevation in two anatomically contiguous leads or $>0.2 \mathrm{mV}$ in leads $\mathrm{V} 1$ 
and V2; iii) elevated levels of MB fraction of creatine kinase or troponin-I at least three times the upper reference limit.

The inclusion criteria of the present study were as follows: i) A diagnosis of acute STEMI and a history of primary PCI, and ii) age of $\geq 65$ years. The exclusion criteria were as follows: i) A Mini-Mental State Examination (MMSE) score of $<15$ before primary PCI, ii) history of drug abuse, iii) history of psychiatric disorders, iv) history of neurologic disease (including stroke), v) previous neuropsychological examination, vi) any severe visual or auditory disorder, vii) Parkinson's disease and viii) unwillingness to comply with the protocol or procedures. The primary PCI of all patients was performed by the same surgical team.

During the enrolment period, a total of 138 patients, who were aged $\geq 65$ years, underwent primary PCI. Among these patients, 27 patients refused to participate this study. Hence, a total of 111 patients with acute STEMI following primary PCI were included in the present study (Table I). All patients enrolled in the present study were initially evaluated by MMSE on the day they were admitted to the hospital.

Measurement of delirium. Patients who were scored as 3-4 (i.e. arousable by verbal stimulation) based on the Richmond Agitation and Sedation Scale were assessed for delirium once daily by trained personnel using the Confusion Assessment Method for the Intensive Care Unit (CAM-ICU) method (8). This can only be used if a patient is arousable by voice without the requirement for physical stimulation. When performed by a well-trained healthcare professional, the CAM-ICU procedure only takes 1-2 min. This method has satisfactory sensitivity and specificity in detecting delirium (9).

The CAM-ICU method evaluates four features of delirium: i) Acute changes or fluctuations in mental state from baseline, ii) inattention, iii) altered consciousness and iv) disorganized thinking. The result of the CAM-ICU was positive if the patient exhibited features 1 and 2, and either feature 3 or 4 . In the present study, patients were assessed by the same psychiatrists within the first four post-operative days (at $8 \mathrm{am}$, daily) (10).

Variables. The following pre-operative characteristics were recorded for each patient: Age, sex, educational level, determination of living alone, body mass index, smoking, history of major depression, history of alcohol abuse (use of three units or more of alcohol daily), history of hypertension, family history of coronary heart disease, history of myocardial infarction, history of PCI, history of diabetes, history of hyperlipidemia, localization of the patient's cardiac infarct (anterior or non-anterior), co-morbidity of arterial fibrillation, co-morbidity of chronic renal insufficiency, co-morbidity of acute left heart failure, co-morbidity of malignant arrhythmia, co-morbidity of electrical defibrillation, co-morbidity of hypotension/cardiac shock, cardiac arrest/cardiopulmonary resuscitation experience, laboratory tests at admission (pre-operative hemoglobin, total cholesterol, low-density lipoprotein, high-density lipoprotein, triglyceride, MB-isoform of creatinine kinase and troponin), peri-operative and post-operative characteristics (length of primary PCI), New York Heart Association classification (NYHA) (11) at $12 \mathrm{~h}$ after the operation, ST-recovery segment at $12 \mathrm{~h}$ after the operation, post-operative mechanical ventilation, intra-aortic balloon pumping (IABP) after the operation, contrast-induced nephropathy, post-operative sleep disorders and post-operative pain [current pain, defined as Visual Analogue Scale/Score (VAS) (12) >4].

Statistical analysis. Statistical analyses were performed using SPSS version 16.0 for Windows (SPSS, Inc., Chicago, IL, USA). Values are expressed as the mean \pm standard deviation or the frequency and percentage. Comparisons were performed using the t-test, Fisher's exact test, Chi-squared test and Mann-Whitney U-test. In the logistic regression analysis, forward stepwise (likelihood ratio) was performed to study the predictors of delirium. For this analysis, the presence or absence of delirium/mortality was introduced as a dependent variable, and all risk factors that differed significantly were introduced as independent variables.

\section{Results}

Incidence of post-operative delirium. The mean age of the patients was $68.95 \pm 12.16$ years. Among the 111 patients, 58 $(52.3 \%)$ were male. Overall, 32 of 111 participants developed delirium, and accordingly, the incidence of delirium after primary PCI in older adults (aged $\geq 65$ years) with acute STEMI was $28.8 \%$.

Risk factors. As presented in Table II, no significant difference was observed between the groups of patients with and without delirium with regard to sex, smoking status, history of major depression, history of coronary heart disease, history of hypertension, history of diabetes mellitus, localization of the cardiac infarct (anterior or non-anterior), co-morbidity of arterial fibrillation and co-morbidity of chronic renal insufficiency. Compared with non-delirious patients, those with delirium were older, had a lower educational level, a lower body mass index and more frequently had a history of alcohol abuse, myocardial infarction, PCI and hyperlipidemia. In addition, patients with delirium more frequently lived alone and had a higher rate of co-morbidity of acute left heart failure, malignant arrhythmia, electrical defibrillation, hypotension/cardiac shock and cardiac arrest/cardiopulmonary resuscitation. The data in Table III indicate that the levels of pre-operative hemoglobin, total cholesterol and low-density lipoprotein in the delirium group were similar to those in the non-delirious group. The participants with delirium had lower levels of high-density lipoprotein and higher levels of triglyceride, MB-isoform of creatinine kinase and troponin (Table III).

The intra-operative and post-operative characteristics are presented in Table IV. Delirious patients had a longer duration of primary PCI and more frequently had sleep disorders and post-operative pain (current pain), when compared with the non-delirious patients. In addition, no significant difference was observed in any of the other parameters, including NYHA at $12 \mathrm{~h}$ after the operation, ST-recovery segment at $12 \mathrm{~h}$ after the operation, post-operative mechanical ventilation and IABP after the operation (Table IV).

The result of the logistic regression indicated that an older age $[\geq 65$ years; odds ratio $(\mathrm{OR})=1.192,95 \%$ confidence interval $(\mathrm{CI})=1.071-1.328, \mathrm{P}=0.001]$, living alone $(\mathrm{OR}=4.827$, 
Table I. Statistical data regarding the patient selection for the present study.

\begin{tabular}{lr} 
Stage & $\mathrm{n}$ \\
\hline Initial enrolment & 138 \\
Exclusion & 16 \\
Primary PCI without screening & 1 \\
Participated in another study & 4 \\
Had no time & 6 \\
Had no interest/refused to participate & 111 \\
Finally included & \\
\hline
\end{tabular}

PCI, percutaneous coronary intervention.

95\% CI=1.315-17.725, P=0.018), history of alcohol abuse $(\mathrm{OR}=3.875,95 \% \mathrm{CI}=1.168-12.857, \mathrm{P}=0.026)$, longer duration of surgery $(>50 \mathrm{~min}$; $\mathrm{OR}=1.152,95 \% \mathrm{CI}=1.077-1.232$, $\mathrm{P}<0.001$ ) and post-operative pain (current pain; $\mathrm{OR}=7.663$, 95\% CI=1.432-41.023, $\mathrm{P}=0.017$ ) were independently associated with post-operative delirium (Table V).

\section{Influence of delirium on hospital outcomes}

Length of hospital stay. Participants who developed delirium had a longer mean hospital stay compared with that of the patients without delirium $(10.2 \pm 1.8$ vs. $6.9 \pm 1.3$ days, $Z=7.172$, $\mathrm{P}<0.001$; Table IV).

Mortality within one year after discharge (one-year mortality). The one-year mortality was similar between patients with and without delirium $[3.1 \%(1 / 32)$ vs. $6.3 \%(5 / 79), Z=0.457$, $\mathrm{P}=0.671$; Table IV].

Readmission within 30 days after discharge. Of the 32 participants with delirium, four were readmitted to the hospital within 30 days $(11 \%)$, while only one of the 79 participants without delirium was readmitted $(1.9 \% ; \mathrm{Z}=4.326, \mathrm{P}=0.038$; Table IV).

\section{Discussion}

Delirium has become a research focus due to its profound consequences, such as longer hospital stay and higher hospitalization expenses (13). In addition, previous studies have demonstrated that understanding the incidence and risk factors of delirium is necessary to improve patient outcomes (14). The reported frequency of delirium ranges widely, depending on the location and type of studies that have investigated delirium. It has been reported that delirium occurs in $20-60 \%$ of patients in Intensive Care Units (15), 18-25\% of oncology patients (16) and 8-36\% of patients undergoing cardiovascular surgery (17). The incidence of delirium after primary PCI in patients with acute myocardial infarction has remained insufficiently investigated. According to a previous study, delirium or agitation were mentioned in physicians' or nurses' progress notes for $28 \%$ of hospitalized patients with acute myocardial infarction (18) in individuals aged $\geq 90$ years. The incidence rate of delirium determined in the present study was $28.8 \%$.
Since the present study was the first study in China to evaluate the incidence of delirium in patients diagnosed with acute STEMI who underwent primary PCI and were within the age group of $\geq 65$ years, the prevalence data of the present study cannot be compared with other studies in China. However, the present result indicates patients aged $\geq 65$ years with acute STEMI and subjected to primary PCI frequently present with delirium.

It is well known that post-operative delirium is an acute neuropsychiatric clinical syndrome primarily characterized by disturbances in memory, thought, behaviour, perception, orientation and consciousness, and mostly occurs within four days after surgery (19). In view of this, delirium assessment was performed by the same psychiatrists within the first four post-operative days in the present study.

The identification of risk factors is critical for implementing prevention efforts. Previous studies on subjects with non-cardiac general surgery have identified older age, alcohol abuse, abnormal electrolytes, thoracic or aortic surgery and poor cognitive or physical functional status as risk factors of delirium (20). In present study, it was indicated that older age, living alone, history of alcohol dependence, longer duration of primary PCI and post-operative pain (current pain) are risk factors for delirium after primary PCI in older patients with acute STEMI. Although the localization of myocardial infarction (anterior or non-anterior) is an important factor for the myocardial damage in subjects with acute myocardial infarction, the present results suggest that the localization of the myocardial infarction (anterior or non-anterior) is not a predictor of delirium after primary PCI, which was similar to the results of a previous study (21). In subsequent studies, more attention will be paid to this factor.

The results of the present study suggest that postoperative pain is an independent risk factor for delirium. Pain is frequently prominent in patients diagnosed with acute STEMI undergoing primary PCI, and pain gives rise to sleep disorders and neuroendocrine disorders, which are prone to causing delirium (22). The present study demonstrated that post-operative pain is an important risk factor for delirium, which requires careful consideration. Therefore, peri-operative pain optimization may be a strategy to reduce the risk of delirium and further research is required to test this hypothesis.

Previous studies have indicated that advanced age is one of the most consistent pre-disposing factors for the occurrence of delirium (23). Consistently, in the present study, advanced age was independently associated with the development of delirium in patients with acute STEMI after primary PCI. The mechanisms may be summarized as follows: First, older age is characterized by the aging of tissues and organs, an increase in the rate of frail subjects and an increased occurrence of cognitive decline and functional impairment (24). Frail elderly patients are more prone to several post-operative complications, including delirium. Second, the aged brain is more susceptible to anaesthetic effects and more sensitive to non-anaesthetic drugs (25). Finally, markers for activated microglia, including major histocompatibility complex class II, increase with aging (26), which may result in an inflammatory response in the brain. A study has also indicated that peripheral infections or pneumonia are closely associated with 
Table II. Sociodemographic and clinical characteristics of patients with and without delirium.

\begin{tabular}{|c|c|c|c|}
\hline Characteristic & $\begin{array}{l}\text { Delirium } \\
(\mathrm{n}=32)\end{array}$ & $\begin{array}{l}\text { No delirium } \\
\qquad(\mathrm{n}=79)\end{array}$ & P-value \\
\hline Male (sex) & $18(56.3)$ & $40(50.6)$ & $0.591^{\mathrm{c}}$ \\
\hline Age (years) & $75.47 \pm 13.16$ & $67.99 \pm 11.27$ & $<0.001^{\mathrm{d}}$ \\
\hline MMSE score & $26.23 \pm 3.42$ & $27.13 \pm 3.11$ & $0.154^{\mathrm{a}}$ \\
\hline Education (years) & $7.06 \pm 4.03$ & $10.2 \pm 4.37$ & $<0.001^{\mathrm{a}}$ \\
\hline Living alone & $10(31.3)$ & $6(7.6)$ & $0.003^{\mathrm{c}}$ \\
\hline Body mass index $\left(\mathrm{kg} / \mathrm{m}^{2}\right)$ & $26.24 \pm 3.37$ & $27.19 \pm 3.08$ & $0.155^{\mathrm{a}}$ \\
\hline History of major depression & $1(3.1)$ & $5(2.5)$ & $0.671^{\mathrm{c}}$ \\
\hline History of smoking & $14(43.8)$ & $32(40.5)$ & $0.753^{\mathrm{c}}$ \\
\hline History of alcohol abuse & $11(34.4)$ & $12(15.2)$ & $0.024^{\mathrm{c}}$ \\
\hline Family history of coronary heart disease & $9(28.1)$ & $19(24.1)$ & $0.654^{\mathrm{c}}$ \\
\hline History of myocardial infarction & $10(31.3)$ & $9(11.4)$ & $0.012^{\mathrm{c}}$ \\
\hline History of percutaneous coronary intervention & $8(25)$ & $5(6.33)$ & $0.014^{\mathrm{b}}$ \\
\hline History of hypertension & $11(34.3)$ & $21(26.6)$ & $0.412^{\mathrm{c}}$ \\
\hline History of diabetes & $10(31.2)$ & $23(29.1)$ & $0.824^{\mathrm{c}}$ \\
\hline History of hyperlipoidaemia & $13(40.6)$ & $15(18.9)$ & $0.017^{\mathrm{c}}$ \\
\hline Localization of the cardiac infarct (anterior) & & & $0.812^{\mathrm{c}}$ \\
\hline Anterior & $17(53.1)$ & $40(50.6)$ & \\
\hline Non-anterior & $15(46.9)$ & $39(49.4)$ & \\
\hline Combined atrial fibrillation & $6(18.8)$ & $13(16.5)$ & $0.771^{\mathrm{c}}$ \\
\hline Combined chronic renal insufficiency & $9(28.1)$ & $17(21.5)$ & $0.457^{\mathrm{c}}$ \\
\hline Combined acute left heart failure & $6(19.4)$ & $3(3.8)$ & $0.026^{\mathrm{b}}$ \\
\hline Malignant arrhythmia & $7(21.9)$ & $5(6.3)$ & $0.04^{\mathrm{b}}$ \\
\hline Electrical defibrillation & $4(12.5)$ & $1(1.3)$ & $0.038^{\mathrm{b}}$ \\
\hline Combined hypotension/cardiac shock & $6(18.8)$ & $2(2.5)$ & $0.01^{\mathrm{b}}$ \\
\hline $\begin{array}{l}\text { Cardiac arrest/cardiopulmonary } \\
\text { resuscitation experience }\end{array}$ & $4(12.5)$ & $1(1.3)$ & $0.038^{\mathrm{b}}$ \\
\hline
\end{tabular}

Values are expressed as $\mathrm{n}(\%)$ or the mean \pm standard deviation. ${ }^{\mathrm{a}}$ Independent-sample t-test; ${ }^{\mathrm{b}}$ Fisher's exact test; ${ }^{\mathrm{c}} \mathrm{Chi}$-squared test; ${ }^{\mathrm{d}} \mathrm{Mann}-\mathrm{Whitney}$ U-test. The parameter which didn't obey normal distribution was analyzed using the Mann-Whitney U-test. The parameters which obeyed a normal distribution were analyzed using a t-test. MMSE, mini mental state examination.

Table III. Laboratory parameters in patients with and without delirium.

\begin{tabular}{|c|c|c|c|}
\hline Parameter & $\begin{array}{l}\text { Delirium } \\
(\mathrm{n}=32)\end{array}$ & $\begin{array}{l}\text { No delirium } \\
\qquad(\mathrm{n}=79)\end{array}$ & P-value \\
\hline Preoperative haemoglobin (g/l) & $136.25 \pm 15.96$ & $134.82 \pm 17.24$ & $0.687^{\mathrm{a}}$ \\
\hline Preoperative total cholesterol (mg/dl) & $176.43 \pm 50.06$ & $163.47 \pm 48.06$ & $0.206^{\mathrm{a}}$ \\
\hline Preoperative low-density lipoprotein (mg/dl) & $107.68 \pm 39.22$ & $99.82 \pm 41.27$ & $0.359^{\mathrm{b}}$ \\
\hline Preoperative high-density lipoprotein (mg/dl) & $33.95 \pm 9.61$ & $38.56 \pm 9.51$ & $<0.001^{\mathrm{a}}$ \\
\hline Preoperative triglyceride (ng/ml) & $122.82 \pm 72.28$ & $91.497 \pm 54.38$ & $<0.001^{\mathrm{b}}$ \\
\hline $\begin{array}{l}\text { Preoperative MB-isoform of creatinine } \\
\text { kinase }(\mathrm{ng} / \mathrm{ml})\end{array}$ & $75.71 \pm 78.13$ & $39.86 \pm 58.87$ & $<0.001^{b}$ \\
\hline Preoperative troponin (ng/ml) & $40.13 \pm 25.78$ & $23.71 \pm 22.36$ & $<0.001^{\mathrm{a}}$ \\
\hline
\end{tabular}

andependent-sample t-test; ${ }^{\mathrm{b}}$ Mann-Whitney U-test. The parameters which didn't obey a normal distribution were analyzed using the Mann-Whitney U-test and those that obeyed a normal distribution were analyzed using a t-test. 
Table IV. Surgical and post-operative characteristics of patients with and without delirium.

\begin{tabular}{|c|c|c|c|}
\hline Item & Delirium $(n=32)$ & No delirium $(\mathrm{n}=79)$ & P-value \\
\hline Length of surgery (min) & $103.8 \pm 23.5$ & $53.4 \pm 22.7$ & $<0.001^{\mathrm{c}}$ \\
\hline NYHA classification $12 \mathrm{~h}$ after operation (score $\geq 3$ ) & $6(18.8)$ & $5(6.3)$ & $0.102^{\mathrm{a}}$ \\
\hline ST recovery segment $12 \mathrm{~h}$ after operation & $10(31.3)$ & $32(40.5)$ & $0.487^{\mathrm{a}}$ \\
\hline Post-operative mechanical ventilation & $3(9.4)$ & $1(1.3)$ & $0.132^{\mathrm{a}}$ \\
\hline Intra-aortic balloon pumping after operation & $1(3.1)$ & $1(1.3)$ & $0.525^{\mathrm{a}}$ \\
\hline Contrast-induced nephropathy & $3(9.4)$ & $3(6.3)$ & $0.475^{\mathrm{a}}$ \\
\hline Sleep disorder & $19(59.4)$ & $23(29.1)$ & $0.003^{\mathrm{b}}$ \\
\hline Post-operative pain [current pain (VAS >4)] & $18(56.3)$ & $21(26.6)$ & $0.003^{\mathrm{b}}$ \\
\hline Length of hospital stay (days) & $10.2 \pm 1.8$ & $6.9 \pm 1.3$ & $<0.001^{\mathrm{c}}$ \\
\hline Mortality within 1 year after discharge $(\%)$ & $1(3.1)$ & $5(6.3)$ & $0.671^{\mathrm{a}}$ \\
\hline Readmission within 30 days after discharge (\%) & $4(11)$ & $1(1.9)$ & $0.038^{\mathrm{a}}$ \\
\hline
\end{tabular}

Values are expressed as $\mathrm{n}(\%)$ or the mean \pm standard deviation. ${ }^{\mathrm{a} F i s h e r}$ 's exact test; ${ }^{\mathrm{b}} \mathrm{Chi}$-squared test; ${ }^{\mathrm{c}}$ Mann-Whitney U-test. The parameters which did not obey a normal distribution were analyzed using the Mann-Whitney U-test and those that obeyed a normal distribution were analyzed using a t-test. NYHA, New York Heart Association; VAS, visual analog scale.

Table V. Predictors of delirium in the study sample (using univariate logistic regression analysis with reference category non-delirious).

\begin{tabular}{|c|c|c|c|c|c|c|}
\hline Factor & $\mathrm{B}$ & S.E. & Wald & P-value & OR & $95 \% \mathrm{CI}$ \\
\hline Age & 0.176 & 0.055 & 10.201 & 0.001 & 1.192 & $1.071-1.328$ \\
\hline Living alone & 1.574 & 0.664 & 5.628 & 0.018 & 4.827 & $1.315-17.725$ \\
\hline History of alcohol abuse & 1.355 & 0.612 & 4.89 & 0.026 & 3.875 & $1.168-12.857$ \\
\hline Length of surgery & 1.127 & 0.625 & 5.32 & $<0.001$ & 1.152 & $1.077-1.232$ \\
\hline Post-operative pain (current pain) & 0.078 & 0.035 & 11.38 & 0.017 & 7.663 & $1.432-41.02$ \\
\hline
\end{tabular}

B, estimated value of the model constant term; S.E., standard error of B; Wald, Wald Chi-square value; CI, confidence interval; OR, is odds ratio.

delirium in elderly people (27). Recently, the proportion of the population aged $\geq 65$ years has rapidly increased (28). Thus, more medical care must be provided for delirium in patients aged $\geq 65$ years.

In recent years, studies have increasingly focused on the influence of social support on the morbidity, mortality and quality of life (29). However, the effect of social support on post-operative delirium has rarely been studied. In the present study, patients who lived alone exhibited a higher risk of developing delirium. A variety of explanations have been offered to account for the association between living alone and delirium. First, isolated patients were reported to have lower incomes and hostility to society in America increased rates of smoking and less social support, compared with those living with company (30). These characteristics may result in delayed medical care for isolated individuals, causing their medical conditions to worsen. In addition, isolated patients may be significantly less likely to be married and have less confidence and receive fewer social visits, which may all contribute to psychological distress. In China, a growing number of elderly individuals live alone and undergo surgery (31), which may lead to an increasing occurrence of post-operative delirium in the elderly. Therefore, more social support should be provided to elderly individuals living alone.

A prolonged operative time was identified as a significant risk factor for delirium in the present study. An increased duration of surgery is usually associated with an increased exposure to microemboli (32) and a systemic inflammatory response. Relevant studies have demonstrated that factors including endotoxins, cytokines and chemokines during the inflammatory process may have a key role in the pathogenesis of delirium $(33,34)$. Therefore, it is likely that the increased duration of primary PCI may predispose patients to post-operative delirium due to increasing the systemic inflammatory response. However, there is currently no consensus regarding the standard or range of the operative time for primary PCI. Therefore, closer attention should be given to this factor in future studies.

In the present study, alcohol abuse appeared to be a significant risk factor for post-operative delirium. The present results may be partly explained by the suppressed immune defence associated with alcoholism and by the inhibited wound healing of chronic alcoholics (35). It should be pointed out that accurate quantitative data for alcohol 
use were not provided by previous studies (36). In a study by Ouimet et al (37), alcohol abuse was defined as the daily use of $>2$ units per day, and was also demonstrated to be a multivariate risk factor for delirium. According to this, in the present study, the daily alcohol dependence was defined as the use of $\geq 3$ units of alcohol daily.

In the present study, the neurocognitive testing of MMSE was performed on the first day of hospitalization and the results were similar for the two groups, indicating a similar pre-operative cognitive function in the two groups. In conclusion, delirium is common after primary PCI in older adults with STEMI, and aged individuals, living alone, history of alcohol dependence, longer duration of primary PCI (>50 min) and severe pain may be associated with a high risk of delirium. Delirium may be associated with a longer hospital stay and higher odds of re-admission. Thus, more effort should be made to prevent delirium in patients after primary PCI, particularly in older adults with acute STEMI.

Of note, the present study has certain limitations. First, the present study was a single-center study, and the present results require to be confirmed through multicenter trials with larger sample sizes. Furthermore, delirium assessment was only performed within the first four post-operative days, and not on all days of each individual's hospitalization. In addition, the occurrence of delirium prior to PCI was not assessed in the present study, and only neurocognitive testing via the MMSE was performed on the first day of hospitalization to assess the pre-operative cognitive function. Similar MMSE testing results in the two groups were considered to indicate a similar pre-operative cognitive function in the two groups, therefore suggesting that this factor had no marked impact on the results. Regarding the influence of delirium on hospital outcomes, only the indexes of readmission within 30 days after discharge and mortality within one year after discharge were used as end-points. Furthermore, the present study didn't observe all complications and comorbidities in the selected patients with acute myocardial infarction, which may have affected the results. A longer assessment may be needed to explore the incidence and risk factors of delirium after primary PCI in older patients (age, $\geq 65$ years) with acute STEMI. In addition, these results may be affected by confounding, but potentially important factors, and in the present study, an adjustment for confounding factors selected $a$ priori was performed based on a literature review (38).

In conclusion, the results of the present study indicated that older patients (age, $\geq 65$ years) with acute STEMI are at a relatively high risk of delirium following primary PCI. Age ( $\geq 65$ years), living alone, history of alcohol dependence, longer length of primary PCI (>50 min) and post-operative pain (current pain) were determined to be risk factors for delirium after primary PCI in the present cohort. Therefore, more attention should be paid to delirium following primary PCI in older patients (age, $\geq 65$ years) with acute STEMI.

\section{Acknowledgements}

The authors gratefully acknowledge the invaluable assistance of the Department of Cardiology, the Third Affiliated Hospital of Anhui Medical University (Hefei, China).

\section{Funding}

No funding was received.

\section{Availability of data and materials}

The datasets generated and/or analyzed during the present study are available from the corresponding author on reasonable request.

\section{Authors' contributions}

GDZ analyzed and interpreted the patient data, JFW analyzed the data, and SL and XHZ were major contributors in writing the manuscript. All authors read and approved the final manuscript.

\section{Ethics approval and informed consent}

The research protocol was in accordance with the guidelines of the World Medical Association's Declaration of Helsinki, and the present study was performed following the approval of the Ethics Committee of the Third Affiliated Hospital of Anhui Medical University (Hefei, China; no. 2014003). All subjects provided written informed consent.

\section{Patients' consent for publication}

Not applicable.

\section{Competing interests}

The authors declare that they have no competing interests.

\section{References}

1. Inouye SK, Westendorp RG and Saczynski JS: Delirium in elderly people. Lancet 383: 911-922, 2014.

2. Moskowitz EE, Overbey DM, Jones TS, Jones EL, Arcomano TR, Moore JT and Robinson TN: Post-operative delirium is associated with increased 5-year mortality. Am J Surg 214: 1036-1038, 2017.

3. Slooter AJ, Van De Leur RR and Zaal IJ: Delirium in critically ill patients. Handb Clin Neurol 141: 449-466, 2017.

4. Marcantonio ER: Delirium in hospitalized older adults. N Engl J Med 377: 1456-1466, 2017.

5. Reddy SV, Irkal JN and Srinivasamurthy A: Postoperative delirium in elderly citizens and current practice. J Anaesthesiol Clin Pharmacol 33: 291-299, 2017.

6. Selnes OA and McKhann GM: Neurocognitive complications after coronary artery bypass surgery. Ann Neurol 57: 615-621, 2005.

7. Shaefi S, Marcantonio ER, Mueller A, Banner-Goodspeed V, Robson SC, Spear K, Otterbein LE, O'Gara BP, Talmor DS and Subramaniam B: Intraoperative oxygen concentration and neurocognition after cardiac surgery: Study protocol for a randomized controlled trial. Trials 18: 600, 2017.

8. Smith HA, Gangopadhyay M, Goben CM, Jacobowski NL, Chestnut MH, Savage S, Rutherford MT, Denton D, Thompson JL, Chandrasekhar R, et al: The preschool confusion assessment method for the ICU: Valid and reliable delirium monitoring for critically ill infants and children. Crit Care Med 44: 592-600, 2016.

9. Khan BA, Perkins AJ, Gao S, Hui SL, Campbell NL, Farber MO, Chlan LL and Boustani MA: The confusion assessment method for the ICU-7 delirium severity scale: A novel delirium severity instrument for use in the ICU. Crit Care Med 45: 851-857, 2017. 
10. Robinson TN, Raeburn CD, Tran ZV, Angles EM, Brenner LA and Moss M: Postoperative delirium in the elderly: Risk factors and outcomes. Ann Surg 249: 173-178, 2009.

11. Apostolakis E and Akinosoglou K: Reexamining the New York heart association functional classification of heart failure. Am J Cardiol 100: 911-912, 2007.

12. Katz J and Melzack R: Measurement of pain. Surg Clin North Am 79: 231-252, 1999.

13. Zhang X, Shao H, Dong X and Qin B: Research advances in ways to evaluate delirium in intensive care unit. Zhonghua Wei Zhong Bing Ji Jiu Yi Xue 30: 381-384, 2018 (In Chinese)

14. Maniar HS, Lindman BR, Escallier K, Avidan M, Novak E, Melby SJ, Damiano MS, Lasala J, Quader N, Rao RS, et al: Delirium after surgical and transcatheter aortic valve replacement is associated with increased mortality. J Thorac Cardiovasc Surg 151: 815-823.e2, 2016.

15. Van Rompaey B, Elseviers MM, Schuurmans MJ, Shortridge-Baggett LM, Truijen S and Bossaert L: Risk factors for delirium in intensive care patients: A prospective cohort study. Crit Care 13: R77, 2009

16. Ljubisavljevic V and Kelly B: Risk factors for development of delirium among oncology patients. Gen Hosp Psychiatry 25: 345-352, 2003.

17. Detroyer E, Dobbels F, Verfaillie E, Meyfroidt G, Sergeant P and Milisen K: Is preoperative anxiety and depression associated with onset of delirium after cardiac surgery in older patients? A prospective cohort study. J Am Geriatr Soc 56 2278-2284, 2008

18. Malone ML, Rosen LB and Goodwin JS: Complications of acute myocardial infarction in patients $>$ or $=90$ years of age. Am J Cardiol 81: 638-641, 1998.

19. Rubino AS, Onorati F, Caroleo S, Galato E, Nucera S, Amantea B, Santini $F$ and Renzulli A: Impact of Clonidine administration on delirium and related Respiratory weaning after surgicalcorrection of acute type-A aortic dissection: Results of a pilot study. Interact Cardiovasc Thorac Surg 10: 58-62, 2010.

20. Rengel KF, Pandharipande PP and Hughes CG: Postoperative delirium. Presse Med 47: e53-e64, 2018

21. Uguz F, Kayrak M, Cíçek E, Kayhan F, Ari H and Altunbas G: Delirium following acute myocardial infarction: Incidence, clinical profiles, and predictors. Perspect Psychiatr Care 46: 135-142, 2010

22. Vaurio LE, Sands LP, Wang Y, Mullen EA and Leung JM: Postoperative delirium: The importance of pain and pain management. Anesth Analg 102: 1267-1273, 2006.

23. Tilouche N, Hassen MF, Ali HBS, Jaoued O, Gharbi R and El Atrous SS: Delirium in the intensive care unit: Incidence, risk factors, and impact on outcome. Indian J Crit Care Med 22: 144-149, 2018
24. Blaum CS, Ofstedal MB and Liang J: Low cognitive performance, comorbid disease, and task-specific disability: Findings from a nationally representative survey. J Gerontol A Biol Sci Med Sci 57: M523-M531, 2002.

25. Magnusson KR, Scanga C, Wagner AE and Dunlop C: Changes in anesthetic sensitivity and glutamate receptors in the aging canine brain. J Gerontol A Biol Sci Med Sci 55: B448-B454, 2000.

26. Godbout JP, Chen J, Abraham J, Richwine AF, Berg BM, Kelley KW and Johnson RW: Exaggerated neuroinflammation and sickness behavior in aged mice following activation of the peripheral innate immune system. FASEB J 19: 1329-1331, 2005.

27. Janssens JP and Krause KH: Pneumonia in the very old. Lancet Infect Dis 4: 112-124, 2004

28. Beck B, Cameron P, Lowthian J, Fitzgerald M, Judson R and Gabbe BJ: Major trauma in older persons. BJS Open 2: 310-318, 2018.

29. Berkman LF, Glass T, Brissette I and Seeman TE: From social integration to health: Durkheim in the new millennium. Soc Sci Med 51: 843-857, 2000

30. Devore EE, Fong TG, Marcantonio ER, Schmitt EM, Travison TG, Jones RN and Inouye SK: Prediction of long-term cognitive decline following postoperative delirium in older adults. J Gerontol A Biol Sci Med Sci 72: 1697-1702, 2017.

31. Feng Z, Falkingham J, Liu X and Vlachantoni A: Changes in living arrangements and mortality among older people in China. SSM Popul Health 3: 9-19, 2016.

32. Caza N, Taha R, Qi Y and Blaise G: The effects of surgery and anesthesia on memory and cognition. Prog Brain Res 169: 409-422, 2008

33. Rudolph JL, Babikian VL, Treanor P, Pochay VE, Wigginton JB, Crittenden MD and Marcantonio ER: Microemboli are not associated with delirium after coronary artery bypass graft surgery. Perfusion 24: 409-415, 2009

34. Girard TD, Pandharipande PP and Ely EW: Delirium in the intensive care unit. Critical Care 12 (Suppl 3): S3, 2008.

35. Diamond T, Stiel D, Lunzer M, Wilkinson M and Posen S: Ethanol reduces bone formation and may cause osteoporosis. Am J Med 86: 282-288, 1989

36. Dasgupta M and Dumbrell AC: Preoperative risk assessment for delirium after noncardiac surgery: A systematic review. J Am Geriatr Soc 54: 1578-1589, 2006

37. Ouimet S, Kavanagh BP, Gottfried SB and Skrobik Y: Incidence, risk factors and consequences of ICU delirium. Intensive Care Med 33: 66-73, 2007.

38. Mu DL, Li LH, Wang DX, Li N, Shan GJ, Li J, Yu QJ and Shi CX: High postoperative serum cortisol level is associated with increased risk of cognitive dysfunction early after coronary artery bypass graft surgery: A prospective cohort study. PLoS One 8: e77637, 2013. 